
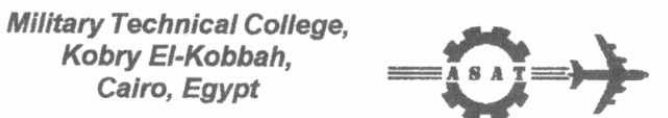

$9^{\text {th }}$ International Conference

On Aerospace Sciences \&

Aviation Technology

\title{
STRESS INTENSITY FACTOR CALCULATION FOR AUTOFRETTAGED TUBE TEST-SPECIMENS USING FINITE ELEMENT METHOD
}

\author{
KANDIL S.A *, ABDEL-KADER M.S"*, MOSTAFA M* and ABDEL WAHAB M.M"
}

ABSTRACT:

The residual stresses, which are generated due to an autofrettage process, in a cracked autofrettaged tube may cause partial crack tip closure. In such a case, the superposition of the stress intensity factor due to both mechanical loading and residual stresses will not produce accurate results. It is, therefore, important to consider the crack closure effect in the calculation of the stress intensity factor. This has been done, in this paper, by using contact elements on the crack faces in order to prevent overlapping. In a first step, the residual hoop stresses for different degrees of autofrettage are calculated from a two-dimensional axisymmetric non-linear elastic-plastic finite element model. Elastic perfectly plastic material behaviour is assumed. The finite element results are in good agreement with the analytical solution found in the literature. For the stress intensity factor calculation, three different autofrettaged test-specimens are considered, namely ring, $\mathrm{C}$-shaped and split ring. Crack length to specimen width ratio between 0.1 and 0.8 and several degrees of autofrettage are considered. Contact elements on the crack faces are used in order to prevent the overlapping and account for any possible partial crack surface closure. A spring and gap in series define the contact element. The stress intensity factor is calculated from the opening displacements behind the crack tip using the two-point displacement formula and the one-point formula.

\section{KEY WORDS}

Autofrettage, stress Intensity Factor, Fracture Toughness, Overstrain, Reverses Yielding, Cold Work and Crack Propagation

*Egyptian Armed Forces.

**Prof. Arab Academy for Science and Technology, Alex. , Egypt

${ }^{\#}$ Assoc. Prof. School of Mech. \& Materials Engineering, University of Surrey, Guildford, UK 


\section{INTRODUCTION}

In order to maximize the efficiency of a thick-walled tube, it must be both durable and lightweight. These criteria apply, among others, to barrels of handguns, rifles, artillery, as well as cannons mounted on military aircrafts. Gun barrel steel has traditionally been used for these purposes because of its relatively high fracture toughness and its ability to develop beneficial residual stresses during autofrettage, despite its rather high density [1]. Autofrettage is carried out by means of an oversized mandrel or swage that is forced through the bore creating a region of plastic deformation. The mandrel is then withdrawn so that the elastic material surrounding the plastic zone attempts to return to its undeformed state. The material that is permanently deformed partially prevents this response. Hence a compressive residual hoop stress field is created about the bore which constrains cracks in this region, if any, to close [2]. Residual stress distribution in thickwalled tubes is a very important factor in design, fracture analysis, and fatigue life estimation. Therefore, this subject matter has met widespread interest of solid mechanics investigators such as Hill [3], Parker et al. [4], Chen [5], Bland [6], Gamer [7] and Megahed and Abbas [8].

The failure of thick-walled tubes is controlled primarily by the fracture toughness, KIC, of the tube material. The larger the KIC, the deeper is the fatigue crack from which the abrupt breakthrough to the outer surface occurs, and the smaller is the length of the breakthrough. This is the preferred fracture mode, a leak-before-peak, in which the final break is small and the tube merely leaks a small amount of the pressurized fluid. A high value of KIC has the equally important effect of delaying or entirely preventing the rapid, unstable fatigue crack growth as the crack approaches the outer surface [9]. Sills and Marmur [2] studied the effect of autofrettage on fracture toughness of thick walled tubes. Tests were carried out on a gun barrel steel to measure KIC under autofrettage conditions of $100 \%$ overstrain.

In this investigation, it is first shown how residual hoop stresses are calculated. The effect of autofrettage on stress intensity factor of thick-walled tubes is then studied. In both cases, analytical and F.E. solutions are obtained and compared.

\section{RESIDUAL HOOP STRESS CALCULATION}

Due to the autofrettage process, residual stresses are introduced in thick-walled tubes. The magnitude and distribution of the residual stresses depend on many factors such as the level of autofrettage, the wall thickness and the yield behaviour of the material. The dimensions and configuration of the cross-section of an autofrettaged tube is shown in Fig. 1. The radius of the internal wall is denoted as $R_{i}$, while that of the outside wall is $R_{0}$. The symbol $R_{\mathrm{s}}$ denotes the radius of the elastic-plastic interface, which is created due to the autofrettage process. The overstrain, $\Phi$, which is a measure of the degree of autofrettage, is defined as:

$$
\Phi=\frac{\mathrm{R}_{\mathrm{s}}-\mathrm{R}_{\mathrm{i}}}{\mathrm{R}_{\mathrm{o}}-\mathrm{R}_{\mathrm{i}}} * 100 \%
$$

The overstrain, $\Phi$, varies between 0 and $100 \%$. At $\Phi=0$, i.e. non-autofrettaged tube, no plastic zone is formed, while at $\Phi=100 \%$, the tube is fully autofrettaged and a complete yielding takes place. 


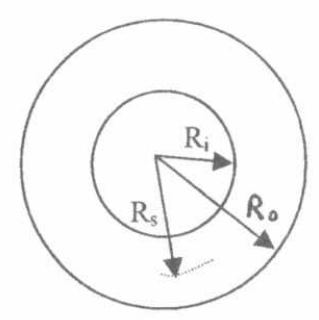

Fig. 1: Cross-section of an autofrettaged tube

A non-linear elastic-plastic finite element model is constructed in order to calculate the residual hoop stresses for different degrees of autofrettage. Due to the axisymmetric nature of an autofrettaged tube, a two-dimensional axisymmetric model is suitable for this analysis. Therefore, a 2-D 8-noded quadrilateral element is used to model the cylinder. The finite element mesh is shown in Fig. 2. For this model, the ratio $R_{0} / R_{i}=2$ was considered with $R_{i}=11 \mathrm{~mm}$. The length of the specimen is of minor importance if it is assumed that the whole cylinder is autofrettaged with the same degree. Therefore a length of about $R_{i} / 2$ was used. A total number of 18 elements through the wall thickness were used as shown in Fig. 2. This number was chosen so that the calculated residual hoop stress converged as a function of element size for a given autofrettage level. The overstrain has been applied to the tube by prescribing the corresponding interference at the inner wall side as initial boundary condition. The interference $\delta$ was calculated using the following equation [8]:

$\delta=R_{m}-R_{i}=\frac{R_{s}^{2}}{R_{i}} \cdot \frac{\sigma_{y}\left(1-v^{2}\right)}{E}$,

where $R_{m}$ is the mandrel radius and $E, v, \sigma_{y}$ are respectively the modulus of elasticity, Poisson's ratio, and yield stress of the material considered. The yielding behaviour used in the analysis is illustrated in Fig. 3 and assumes elastic perfectly plastic material response.

The solution was obtained in several appropriate steps. The prescribed interference was applied gradually until it reaches its maximum value; then it was removed gradually in order to simulate the unloading process. The automatic time stepping, which is available in the finite element package ANSYS, was used. This feature allows the program to optimise the substep sizes according to the progress in the solution. A contour plot of the residual hoop stress is shown in Fig. 4.a for the cases of $60 \%$ overstrain. Moreover, the distribution of the residual hoop stress is plotted with radial distance in Fig. 4.b. 


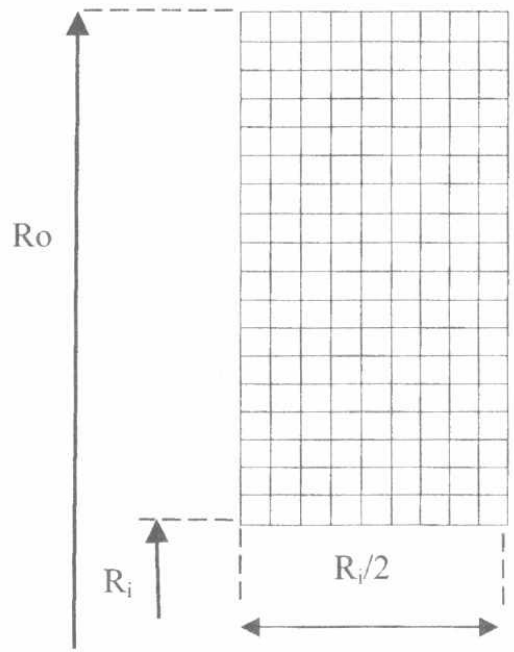

Fig. 2: FE mesh: Axisymmetric model

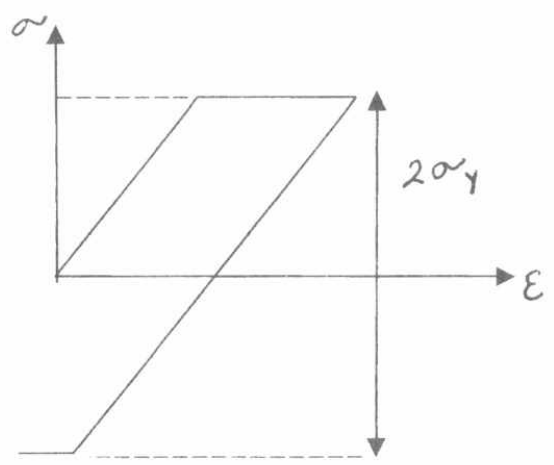

Fig. 3: Yielding behaviour of barrel material used. 

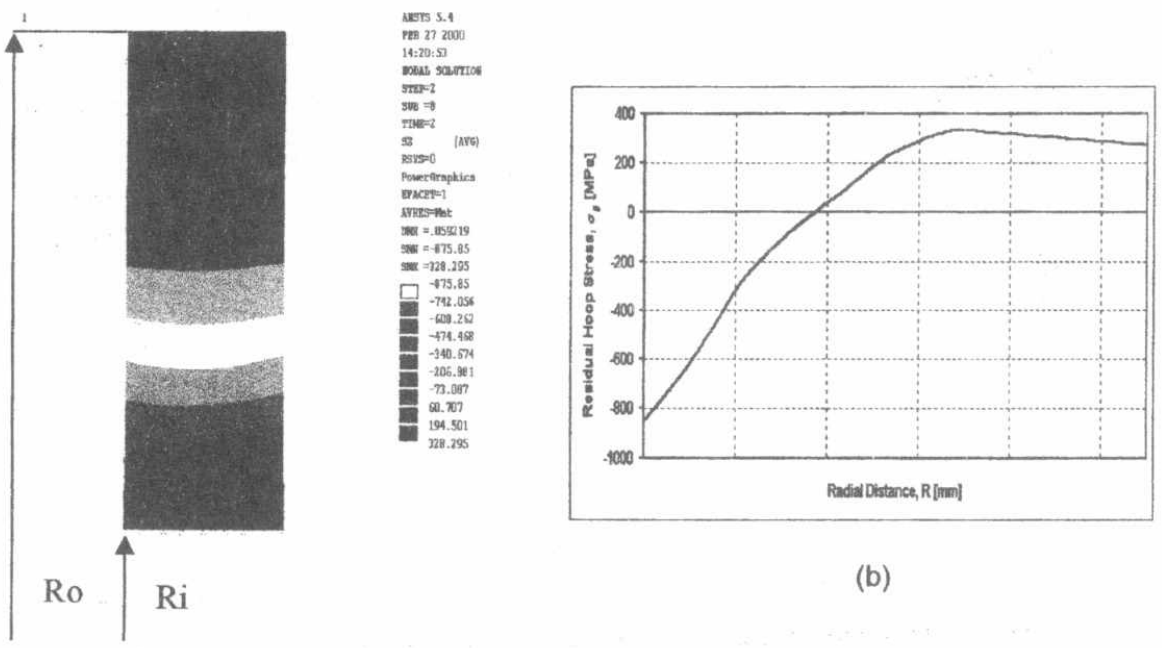

(b)

(a)

Fig. 4: Residual hoop stress in an autofrettaged tube (finite element results):

(a) contour plot, (b) distribution with radial distance.

According to Hill [3], the analytical solution of the residual hoop stress $\sigma_{\theta}$ for an elastic perfectly plastic material can be obtained as:

$\sigma_{\theta}= \begin{cases}-p^{*}+\sigma_{y}\left(1+\ln \frac{r}{R_{i}}\right)-\frac{P^{*} R_{i}^{2}}{R_{o}^{2}-R_{i}^{2}}\left(1+\frac{R_{o}^{2}}{r^{2}}\right) & \text { for } \mathrm{R}_{\mathrm{i}} \leq r \leq \mathrm{R}_{\mathrm{s}} \\ \left(\frac{\sigma_{y} R_{s}^{2}}{2 R_{o}^{2}}-\frac{P^{*} R_{i}^{2}}{R_{o}^{2}-R_{i}^{2}}\right)\left(1+\frac{R_{o}^{2}}{r^{2}}\right) & \text { for } \mathrm{R}_{\mathrm{s}} \leq r \leq \mathrm{R}_{\mathrm{o}}\end{cases}$

where the autofrettage pressure, $\mathrm{p}^{*}$, is interpolated between the pressure for initial yielding, $\mathrm{p}_{\mathrm{i}}$, and the pressure for complete yielding, $\mathrm{p}_{\mathrm{o}}$, which are given by:

$p_{i}=\frac{\sigma_{y}}{2 R_{o}^{2}}\left(R_{o}^{2}-R_{i}^{2}\right)$,

$p_{o}=\sigma_{y} \ln \left(\frac{R_{o}}{R_{i}}\right)$,

The FE and analytical solutions obtained for the analysed present barrel are shown in Fig. 5 for three different overstrain values, namely $\Phi=25,60$ and $100 \%$. Both solutions are seen to provide qualitatively similar results, although discernible differences do exist, particularly in the plastic zone. In the remainder of this paper, only the overstrain $\Phi=60 \%$ will be considered, since it gave better match between FE and analytical results when compared to $\Phi=25 \%$. On the other hand, $\Phi=100 \%$ is hardly considered in barrel manufacture. 


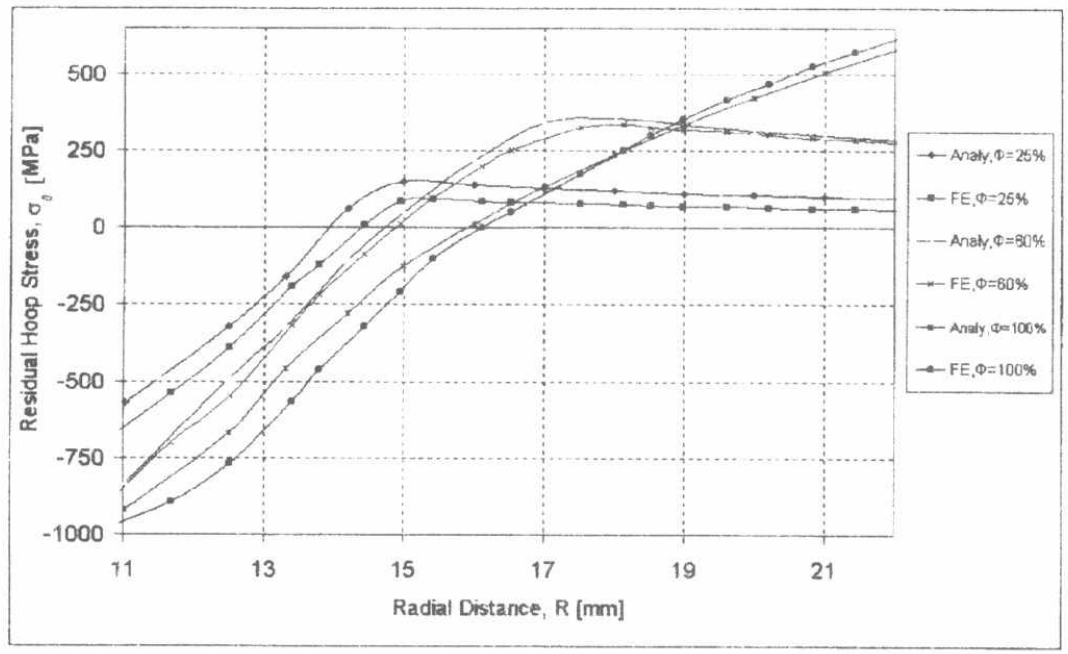

Fig. 5: Finite element and analytical residual hoop stress versus radial distance for different overstrains, $\Phi=25 \%, 60 \%$ and $100 \%$.

\section{FRACTURE TOUGHNESS CALCULATION}

\subsection{Stress Intensity Factor Calculation.}

Three different autofrettaged test-specimens are considered in this study, namely ring, C-shaped and split ring. These test-specimens are widely used for fatigue crack growth tests and therefore the stress intensity factor that takes into account the residual stresses due to the autofrettage process is required. The specimens dimensions and configurations are shown in Fig. 6 [11].

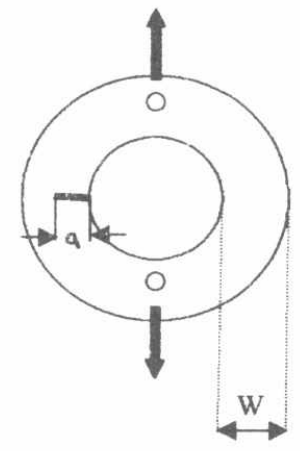

(a) Ring specimen

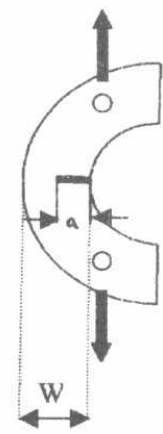

(b) C-shaped specimen

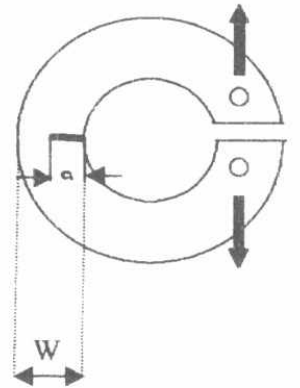

(c) Split-ring specimen

Fig. 6: Test-specimens configurations 
The stress intensity factor is calculated from the opening displacements behind the crack tip. The opening displacement $u_{y}$ for the crack shown in Fig. 7 is given by [9]:

$u_{y}=\frac{K_{I}}{\mu} \sqrt{\frac{r}{2 \pi}} \sin \frac{\theta}{2}\left(2-2 \kappa-\cos ^{2} \frac{\theta}{2}\right)+\frac{K_{\mu}}{\mu} \sqrt{\frac{r}{2 \pi}} \cos \frac{\theta}{2}\left(-1+2 \kappa-\sin ^{2} \frac{\theta}{2}\right)$

where $K_{1}, K_{\|}$are the stress intensity factors for modes I and II, respectively. The local co-ordinates $r$ and $\theta$ are defined in Fig. 7 and $\mu$ is the shear modulus. The constant $k$ is related to Poisson's ratio, $v$, through the relationships: $k=v$ for the plane strain state and $k=v /(1+v)$ for the plane stress state.

For the opening displacement behind the crack tip, $\theta=180^{\circ}$, equation (5 becomes:

$u_{y}=\frac{2 K_{I}}{\mu} \sqrt{\frac{r}{2 \pi}}(1-\kappa)$,

Which reduces to:

$$
u_{y}=\frac{4 K_{I}}{E} \sqrt{\frac{r}{2 \pi}}
$$

For isotropic materials, $\mu=E / 2((1+v)$, under the plane stress condition.

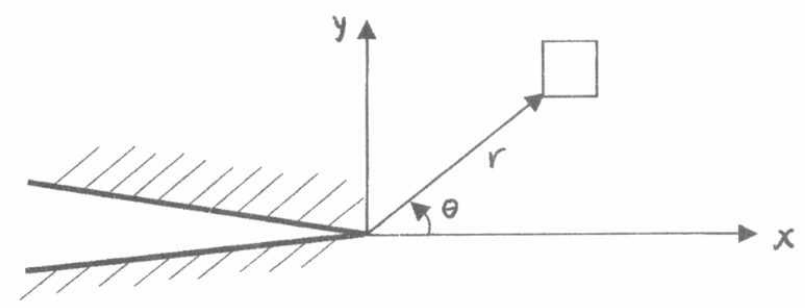

Fig. 7: Local co-ordinate systems near a crack tip

\section{Two-point formula}

For the nodes located behind the crack tip in the singularity element as shown in Fig. 8 , the displacement along the crack face is expressed in terms of the nodal displacements as [9]:

$u_{y}=u_{y_{i}}+\left(-u_{y_{i-2}}+4 u_{y_{i-1}}-3 u_{y_{i}}\right) \sqrt{\frac{r}{L}}+\left(2 u_{y_{i-2}}-4 u_{y_{i-1}}+2 u_{y_{i}}\right) \frac{r}{L}$ 


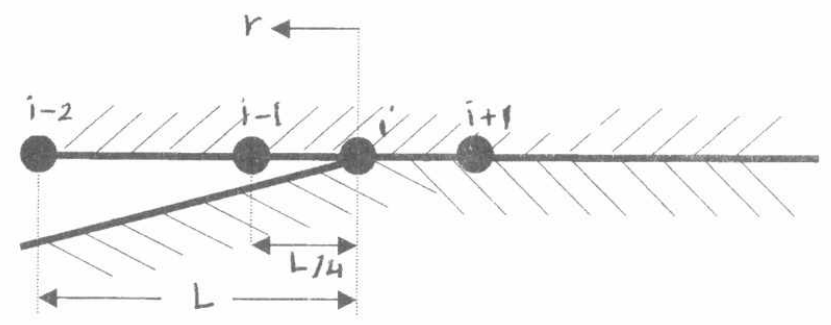

Fig. 8: Crack face nodes in a singularity element

Where $u_{y_{i-2}}, u_{y_{i-1}}, u_{y_{i}}$ are the nodal opening displacements.

For a symmetric crack, $u_{y_{i}}=0$, equation (8) yields,

$u_{y}=\left(4 u_{y_{i-1}}-u_{y_{i-2}}\right) \sqrt{\frac{r}{L}}+\left(2 u_{y_{i-2}}-4 u_{y_{i-1}}\right) \frac{r}{L}$

Comparing equation (7) to equation (9) and equating the terms of $\sqrt{r}$ in both equations, yields:

$K_{I}=\frac{E}{4} \sqrt{\frac{2 \pi}{L}}\left(4 u_{y_{i-1}}-u_{y_{i-2}}\right)$

Equation (10) is known as the two-point displacement formula, since two nodal displacements are required to compute the stress intensity factor.

\section{One-point formula}

From equation (7), if the displacement $u_{y}$ is put equal to $u_{y_{-1}}$ at $\mathrm{r}=\mathrm{L} / 4$, the stress intensity factor will be equal to:

$K_{I}=\frac{E}{2} \sqrt{\frac{2 \pi}{L}} u_{y_{i-1}}$

Again, equation (11) is called the one-point formula as only one nodal displacement is used to compute the stress intensity factor. The one-point formula is in general more accurate than the two-point formula, especially for large crack tip element size. This is because the theoretical square root displacement variation at the crack tip, which has been approximated using singularity elements, is only true very close to the crack tip. In the present analysis, both formulae are used to compute the stress intensity factor. The crack tip element size is decreased until the difference between the two values is less than $1 \%$. This assures the convergence of the results. 


\subsection{Finite Element Considerations.}

Finite element models for the specimens shown in Fig. 6 were constructed using 8 -noded quadrilateral elements. Due to symmetry, only half of each specimen needed to be modelled. The axis of symmetry is the horizontal line that cuts the specimens through the crack. Plane stress condition was assumed for the analysis since the thickness of the specimens was small compared to the wall thickness. The finite element mesh for the ring and the split-ring specimens is the same; however, each specimen has different boundary conditions. The ratio of the crack length to the specimen width, $\mathrm{a} / \mathrm{W}$, can vary between 0.1 to 0.9 . The FE mesh for the C-shaped specimen is shown in Fig. 9 and that for the ring and the split-ring in Fig. 10, for the case of $a / W=0.5$. At the crack tip, singularity elements are used, in which the mid-side nodes are shifted to the quarter position in order to produce square-root stress singularity [13]. Modelling the stress singularity in this way will avoid the use of extensive mesh refinement of standard elements in order to achieve accurate results. The crack tip element size was selected to be at least $\mathrm{a} / 8$. Decreasing the crack tip element size was used to check the convergence of the results. The FE idealisation at the crack tip is shown in Fig. 11. The 8-noded quadrilateral elements were collapsed to 6-noded triangular elements at the crack tip. These triangular elements were expected to produce more accurate results than the quadrilateral elements.

Two different methods were used in order to study the stress intensity factor, namely superposition and contact elements. The superposition method states that the stress intensity factor due to external loading is equal to that due to stresses acting at the crack faces. This argument is also applicable in case of residual stresses. Consider the residual stress distribution in an uncracked body as shown in Fig. 12a. The stress intensity factor in this case is obviously equal to zero, $K_{a}=0$, as there is no crack. In case of a crack, Fig. $12 \mathrm{~b}$, a negative stress, $\mathrm{Y}$, is required on the crack faces in order to keep the stress intensity factor zero, i.e. $K_{b}=0$. The case $b$ can then be separated into case c, stress on the crack lips, and case d, stress on crack faces. This means that the stress intensity factors of cases $c$ and $d$ are equal but opposite in sign. Thus, in the finite element analysis the residual hoop stresses due to the autofrettage are directly applied to the crack faces [12]. From Fig. 12 it can be seen that the stress intensity factor due to the external loading $\left(\mathrm{K}_{\mathrm{e}}\right)$ is superimposed on that due to the residual stresses $\left(\mathrm{K}_{\mathrm{r}}\right)$. This means that if the residual stresses on the crack faces are larger than the applied external loading, e.g. in case of high autofrettage level, the crack faces will overlap. In such a case, the total stress intensity factor $\left(K_{e}+K_{r}\right)$ will be negative, which has no physical meaning. In practice, however, partial crack closure will take place. In the finite element model, this approach is modelled by applying the residual stresses and external load and allowing the crack faces to overlap.

In contrast to the superposition approach, the use of contact elements on the crack faces will prevent the overlapping and account for any partial crack closing. The contact element is defined by a spring and gap in series. If the force in the spring is compression the gap closes, while if the force is tension the gap opens. In the next section, more detailed results and comparison between the superposing of $\mathrm{K}$ and the use of contact elements will be given. 


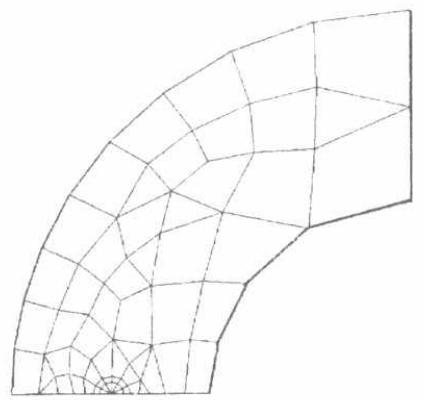

Fig. 9: Finite element mesh for the C-shaped specimen

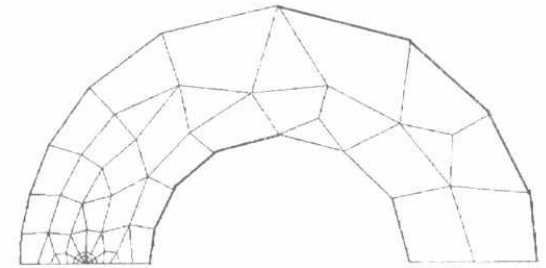

Fig. 10: Finite element mesh for the ring and split-ring specimens

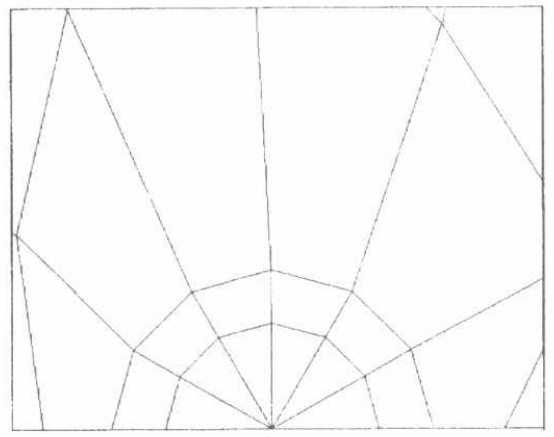

Fig. 11: Finite element idealisation at the crack tip 


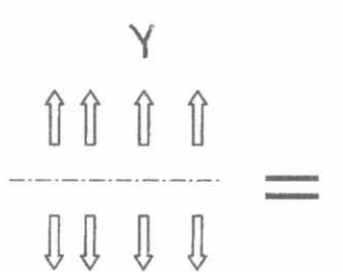

(a) $K_{a}=0$

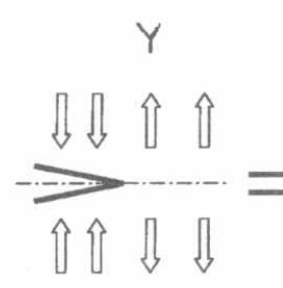

(b) $K_{b}=0$

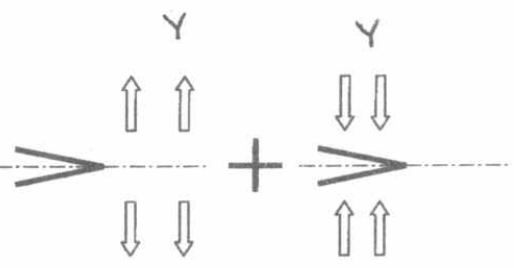

(c) $\mathrm{K}_{\mathrm{c}}$

(d) $k_{d}$

Fig. 12: Superposition of loading

\section{RESULTS AND DISCUSSION:}

Fig.13 depicts the development of the stress intensity factor during loading for the autofrettaged ring specimen $(\Phi=60 \%)$ using the contact elements method, Fig. 13(a), and the superposition method, Fig. 13(b). In both cases, different length to width ratios were considered, which ranged from 0.1 to 0.8 . As may be seen from both Fig.s, the stress intensity factor increases monotonically with the load applied. In general, both methods yielded similar responses, although some differences do exist; the response shown in Fig. 13(a) is bilinear, where as that of Fig. $13(b)$, is quasi-linear. For $a / w=0.1$, for instance, $K$ will not be able to develop before the load attains a value greater than $8 \mathrm{kN}$ and builds up to $\sim 30$ MPam $^{1 / 2}$ when the load increases to $20 \mathrm{kN}$, Fig. 13(a). When the superposition method is used, Fig. 13(b), K starts to develop only when the load is increased above $10 \mathrm{kN}$, although it attains the same value, $30 \mathrm{MPam}^{1 / 2}$, at $\mathrm{F}=20 \mathrm{kN}$. On the other hand, for $\mathrm{a} / \mathrm{w}=0.8, \mathrm{~K}=96 \mathrm{MPam}^{1 / 2}$ is ultimately obtained at $\mathrm{F}=20 \mathrm{kN}$ for both methods, although the $K$ values are considerably different in the low load level. The same responses shown in Fig. 13 were also cross-plotted in Fig. 14 to depict the variation of the stress intensity factor with crack length to width ratio for different load levels. It is clear that at the low levels of loading both methods yielded different responses. The differences diminish as the load increases until they disappear at $\mathrm{F}=20 \mathrm{kN}$.

Figs. 15(a), (b) and 16(a), (b) show respectively the variation of the stress intensity factor with crack length to width ratio for different load levels for the autofrettaged $(\Phi=60 \%)$ split ring and C-shaped specimens. Generally, the responses obtained are similar to those of Figs. 14(a), (b) and the analysis pertaining to Figs. 13 and 14 holds also for Figs. 15 and 16 except that values of the stress intensity factor are much higher than those obtained for the ring specimen. For instance, the C-shaped specimen yields $\mathrm{K}=139 \mathrm{MPa} \cdot \mathrm{m}^{1 / 2}$ corresponding to $\mathrm{F}=10 \mathrm{kN}$ and $\mathrm{a} / \mathrm{w}=0.8$, whereas the split ring specimen yields $\mathrm{K}=147 \mathrm{MPa} \cdot \mathrm{m}^{1 / 2}$ under the same loading and crack length to width ratio. Another important observation is that the responses in Fig. 14 are more or less quasi-linear, whereas those of Figs. 15 and 16 curve up. 
It is also important to note that the comparison between the three geometries should be made at the same conditions. For instance, at $F=10 \mathrm{kN}$ and $\mathrm{a} / \mathrm{w}=$ $0.8, \mathrm{~K}=54 \mathrm{MPa} \cdot \mathrm{m}^{1 / 2}$ for the ring specimen, $147 \mathrm{MPa} \cdot \mathrm{m}^{1 / 2}$ for the split ring specimen and $139 \mathrm{MPa} \cdot \mathrm{m}^{1 / 2}$ for the C-shaped specimen. In other words, the stress intensity factor for the $\mathrm{C}$-shaped specimen and the split ring specimen are very close to each other. While for the ring specimen it is less than half of the above value.

\section{CONCLUSIONS:}

Stress intensity factors have been calculated using two formulas which are two point formula, one point formula for cracked C-shaped, split-ring, and ring test specimen. In both formulas singularity elements were used and $\mathrm{K}$ was calculated with the rate of change of crack length.

Comparisons of the $\mathrm{K}$ calibrations obtained numerically, using finite elements method have indicated good agreement with other information in the literature. It is shown that the formula quoted for C-shaped specimens in the ASTM standard for fracture toughness test is valid for the split-ring geometry examined in this investigation.

Fatigue crack growth studies have revealed that any of the three specimen shapes is suitable for establishing the fatigue crack growth properties in the radial direction of thick-walled tube materials. The ring geometry is suitable for determining the behavior of cracks subjected to fatigue in the presence of residual stresses introduced by autofrettage since manufacture of this shape will not cause any change in the residual stress distribution. 


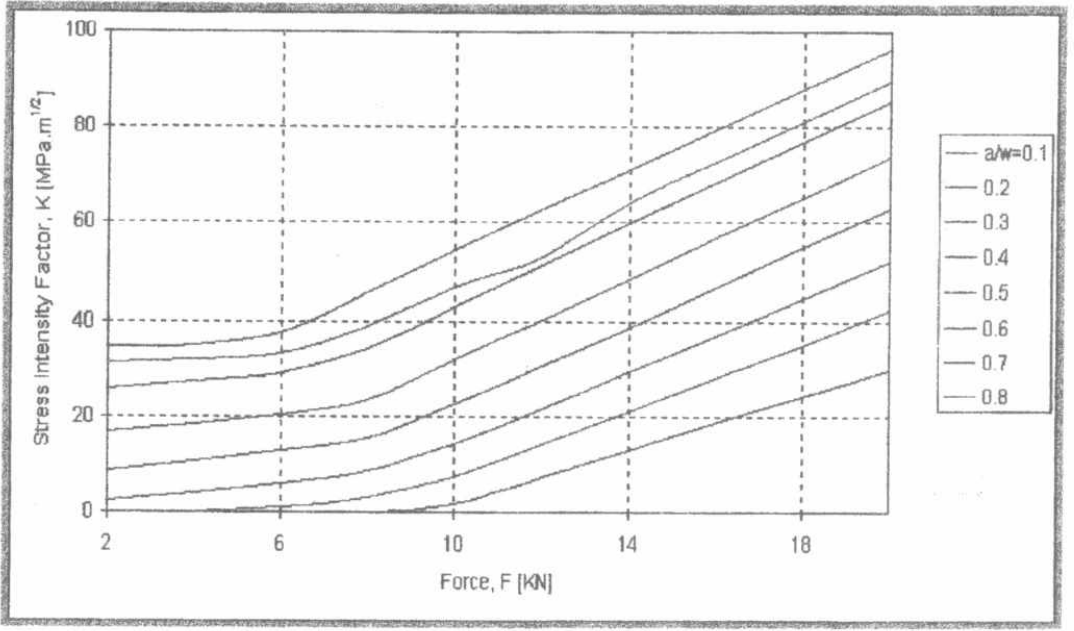

(a)

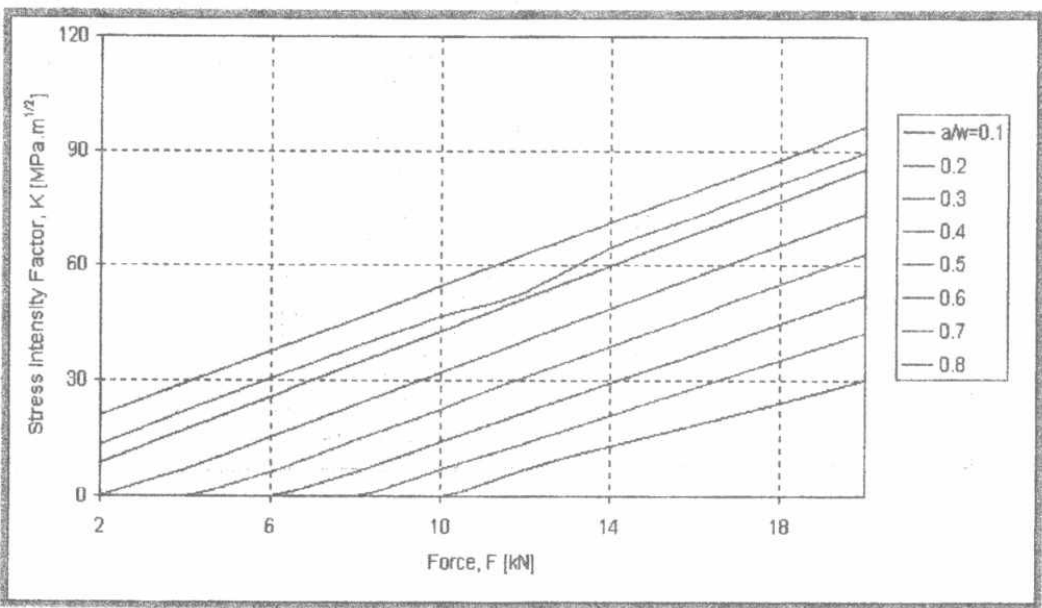

(b)

Fig. 13: Stress intensity factor versus force for autofrettaged ring specimen with $\Phi$ $=60 \%$ applying different crack length to specimen thickness ratio: $\mathrm{a} / \mathrm{w}=0.1-0.8$ a. Using contact element method,

b. Using superposition method. 


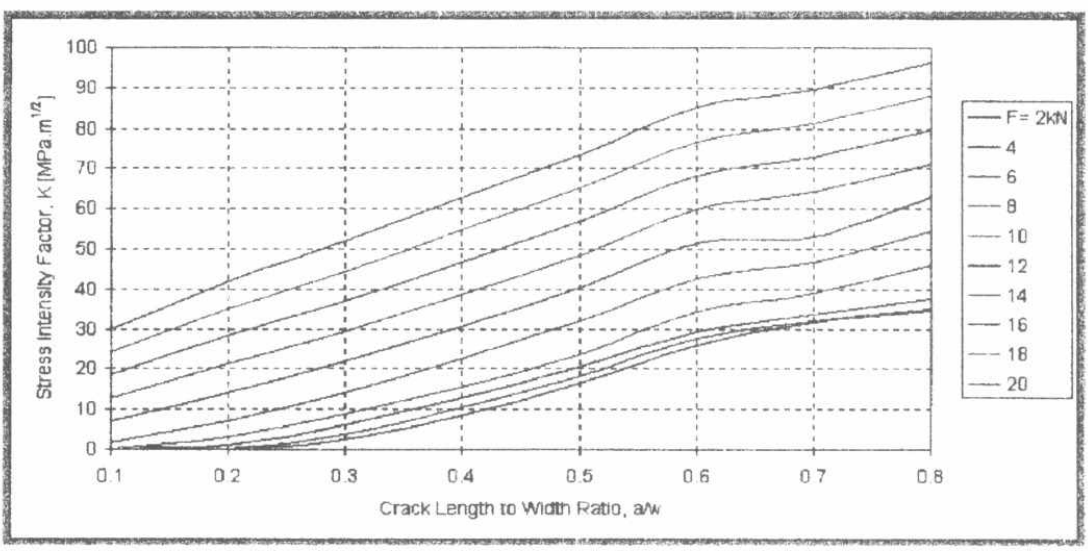

(a)

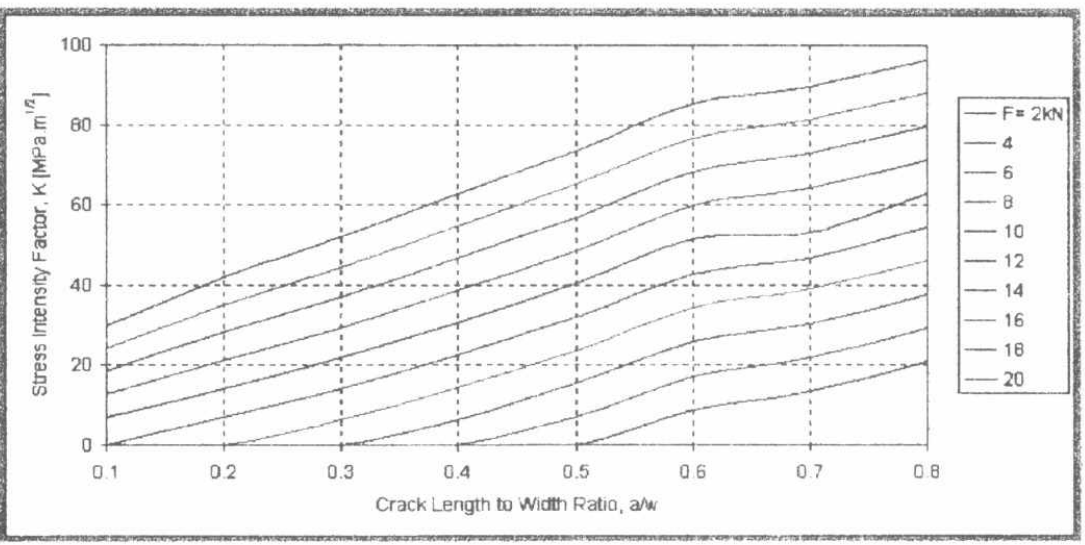

(b)

Fig. 14: Stress intensity factor versus crack length for autofrettaged ring specimen with $\Phi=60 \%$ applying different force values from $F=2 \mathrm{KN}$ to $F=20 \mathrm{KN}$,

a. Using contact element method,

b. Using superposition method. 


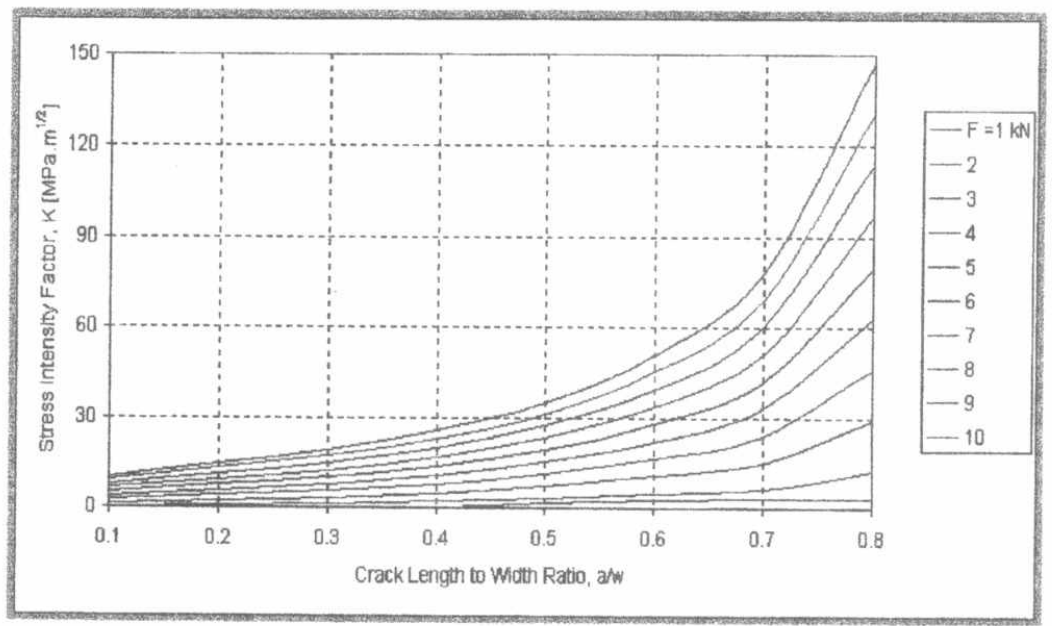

(a)

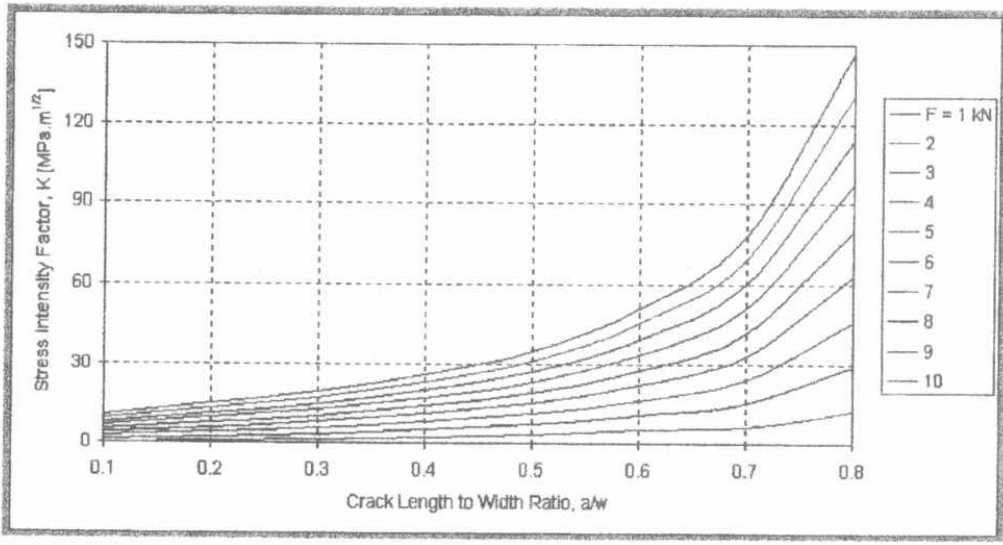

(b)

Fig. 15: Stress intensity factor versus crack length for autofrettaged split-ring specimen with $\Phi=60 \%$ applying different force values from $F=1 \mathrm{KN}$ to $\mathrm{F}=10 \mathrm{KN}$,

a. Using contact element method,

b. Using superposition method. 


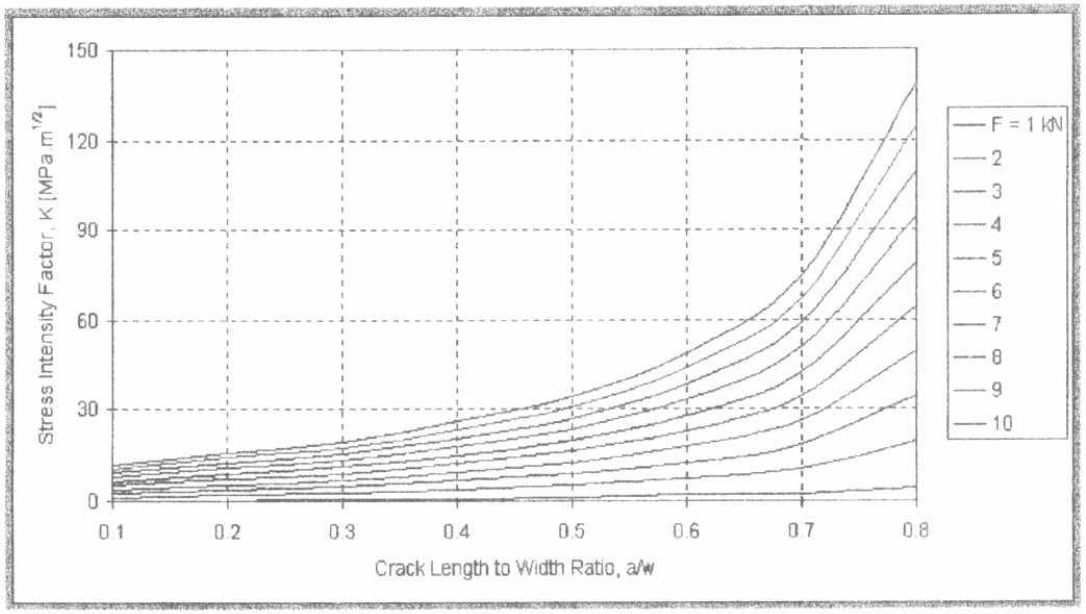

(a)

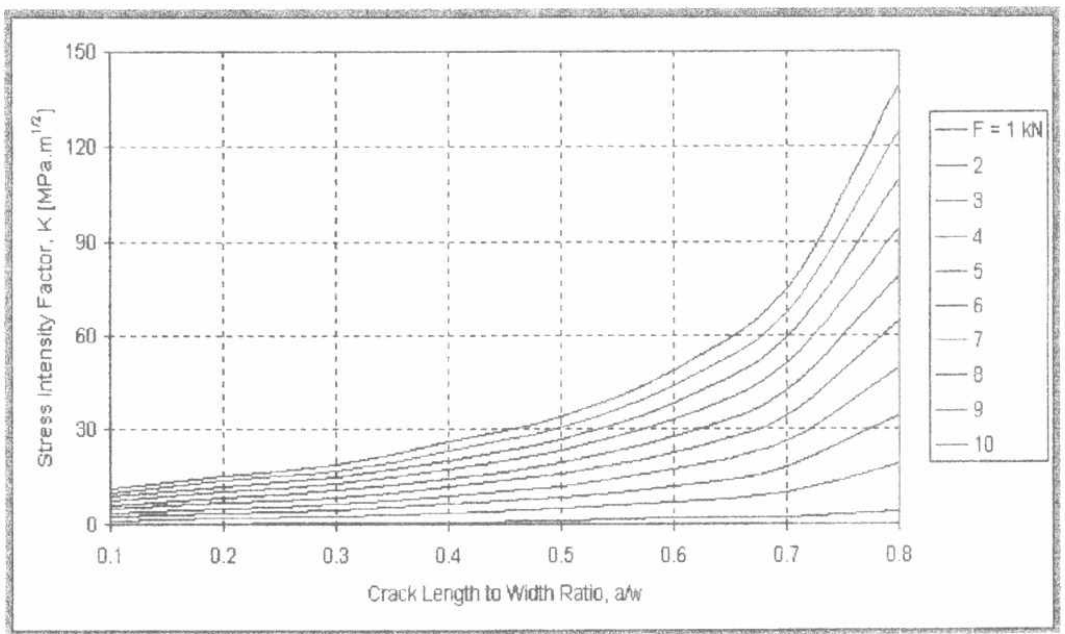

(b)

Fig. 16: Stress intensity factor versus crack length for autofrettaged C- shape specimen with $\Phi=60 \%$ applying different force values from $F=1 \mathrm{KN}$ to $\mathrm{F}=10 \mathrm{KN}$, a. Using contact element method,

b. Using superposition method. 


\section{REFERENCES:}

[1] R.S. Salzar, 1999, "Influence of Autofrettage on Metal Matrix Composite Reinforced Gun Barrels" Composites: Part B 30, pp.841-847

[2] L. B. Sills and I. Marmur, 1989, "Influence of Autofrettage on Fracture Toughness" ,Int. J. of Fracture, Vol. 40, pp. 143-155.

[3] R. Hill, 1967, "The Mathematical Theory of Plasticity", Oxford University Press.

[4] A. Parker, J Underwood, J. Throop, and C Andrasic,1983, "Stress Intensity and Fatigue Crack Growth in a Pressurized, Autofrettaged Thick Cylinder", ASTM STP 791, American Society for Testing and Materials, Philadelphia, pp. I-216 I-237.

[5] P. Chen, 1986, "The Bauschinger and Hardening Effect on Residual Stress in an Autofrettaged Thick- Walled Cylinder", Journal of Pressure Vessel Technology, Vol. 108, pp. 108-112.

[6] D. Bland, 1956, "Elastoplastic Thick-Walled Tubes of Work-Hardening Material Subject to Internal Pressure and to Temperature Gradient", J. Mech. Phys. Solids Vol. 4, pp. 200-205.

[7] U. Gamer, 1988, "The Expansion of Elastic Plastic Spherical Shell with NonLinear Hardening" ,Int. J. Mech. Sci., Vol. 30, pp. 415-426.

[8] M. M. Megahed and A. T. Abbas, 1991, "Influence of Reverse Yielding on Residual Stress Induced by Autofrettage" , Int. J. Mech. Sci., Vol. 33, pp. 139150.

[9] J. Martinez, and J. Dominguez, 1984 "Short Communication on the Use of Quarter-Point Boundary Elements for Stress Intensity Factor Computations" , Int. J. Num. Meth. Engn., Vol. 20, pp. 1941-1950.

[10] Y.I. El-shaer, M. S. Abdel-Kader, M. M. El-Maddah, And M. .M. Megahed, 2000, "Experimental and Analytical Investigations of Residual Stress Induced by Autofrettage", Seventh Cairo University International MPD Conference, pp. 195204.

[11] G. A. Webster, G. C. Klintworth and A. Stacey, 1983, "Stress Intensity Factors for Cracked C-Shaped and Ring Type Test-Pieces", J. of Strain Analysis, Vol. 18, No.4, pp. 225-230.

[12] X. B. Lin and R. A. Smith, 1997, "Stress Intensity Factors for Semi-Elliptical Internal Surface Cracks in Autofrettaged Thick-Walled Cylinders", J. of Strain Analysis, Vol. 32, pp. 351-363.

[13] R.S. Barsoum, 1976, "On the Use of Isoparametric Finite Elements in Linear Elastic Fracture mechanics", Int. J. Num. Meth. Engn., Vol. 10, pp. 25-37. 